\title{
In Vitro Efficacy Assessment of Some Toothpastes and Miswak against Streptococcus Mutans
}

\author{
Arwa Hussain Ali Al-Onayzan ${ }^{1}$, Noura Abdulrahman Hussain Almulhem ${ }^{2}$, \\ Njood Saleh Abdullah AlAboud ${ }^{3}$, Haneen Hamad Sultan Al-Sultan ${ }^{4}$, Sayed Ibrahim Ali $^{5}$, \\ Naglaa Ali Hassan Sidiq ${ }^{6}$ \\ ${ }^{1,23,4}$ Medical students at King Faisal University, College of Medicine, Saudi Arabia \\ ${ }^{5,6}$ King Faisal University, College of Medicine, Saudi Arabia
}

\begin{abstract}
Background: Tooth decay is a common chronic childhood disease of children aged 5 to 17 years. Streptococcus mutans (S muutans) is one of the causative agents. It is a preventable disease by oral hygiene, which include toothpastes and Salvadora persicain (Miswak). Objective: This study evaluates the effect of some toothpastes and Salvadora persicain on S mutans microorganism. Methods: AloeDent ${ }^{\circledR}$ and Crest ${ }^{\circledR}$ toothpastes were tested against $S$ mutans on Muller Hinton agar (MHA). Followed by testing of Miswak against the same bacteria in three different preparations. Results: Crest toothpaste showed minimal effect against the bacteria. Miswak tests gave significant results. Conclusion: Miswak can be proposed as a good natural preventive anti $S$ mutans agent. Toothpastes need attention and investigation by Saudi food and drug authority.
\end{abstract}

Keywords: Streptococcus mutans, Miswak, toothpastes

\section{Introduction}

Tooth decay and periodontal diseases are one of the most common bacterial infections, interestingly; dental caries are ranked the second of the most prevalent diseases after cold in humans [1-3]. In addition, it is considered the most common chronic childhood disease of children aged 5 to 17 ; five times more common than asthma and seven times more than hay fever $[2,4]$.

Dental plaque is known as the major beginning factor for dental caries and periodontal diseases and it is formed by the accumulation of microorganisms on the tooth surface as a biofilm[1, 5, 6]. Among the wide variety of oral microflora $S$ mutans are the main organisms that are found in the plaque[6-9]. The pathogenicity of S.mutans is explained by its strong affinity for the tooth surface and its other virulence factors such as their ability to synthesize extracellular polysaccharides from sucrose, using glucosyltransferase which explains the increased chance of caries formation in sucrose-rich diet [6].

Many methods are used to control the dental plaque and to maintain oral hygiene including the use of Miswak chewing sticks, chemical methods and toothpastes[10]. Wide variety of compounds are found in different toothpastes, such as, triclosan, chlorhexidine and particularly fluoride, which have demonstrated clinical efficacy in controlling dental plaque $[11,12]$. In Middle East countries such as Saudi Arabia, there is a trend toward using Miswak chewing stick as a toothbrush. The miswak chewing sticks are derived from plant called Salvadora persica, a member of Salvadoraceaefamily, which is found in Africa, South America and theMiddle East [10]. Miswak has been found to contain antibacterial properties against cariogenic bacteria which has an ability to disrupt plaque accumulation[10,13,14]. It was also recommended by world health organization as an effective oral hygiene tool $[15,16]$. Since the S.mutans is strongly related to dental plaque[1,6], this experimental study aims to assess the efficacy of some toothpastes along with the efficacy of Miswak against S.mutans. It will be a helpful step, highlighting the natural antibacterial properties of miswak.

\section{Materials and Methods}

An experimental study that was conducted in King Faisal University (KFU) lab and were approved by our Ethics Committee. Materials collected from local markets and a farm in Al Ahssa in KSA.

\section{Materials}

Six brands of toothpaste were randomly purchased from the market in Al-Ahssa, KSA; AloeDent ${ }^{\circ}$, Crest $\AA$, Colgate $\AA$, Signal®, Closeup®, Siwak.F® and Children Siwak.F®. Salvadora Persica SP (Miswak) was provided from a farm in Umran village in a powder form. S mutans was a lab strain; unknown. Blood agar (BA) Columbia scharlau 01-034, MacConkey agar (MCA) Scharlau 01-118 and MuellerHinton agar (MHA) Medical science co.Itd QMSIS3PLISO, are the media been used. Methanol-99.9\% ( $\mathrm{MeOH})$, normal saline and Dimethyl sulfoxide (DMSO) were used. $37 \mathrm{C}^{\circ}$ incubator and Soxhlet extractor were the equipment is available.

\section{Testing of toothpastes}

The toothpastes were tested for possibility of contamination in BA and MCA. Four of them were excluded and only two were used Crest ${ }^{\circledR}$ and AloeDent ${ }^{\circledR}$.

\section{Collection of chewing sticks}

The fresh roots were dried, powdered and kept in sterile screw-capped bottles.

\section{Preparation of Miswak extract}

One gram (gm) of Miswak powder was mixed with $10 \mathrm{ml}$ of normal saline and kept soaked for 5 minutes. Then, sterilized paper disks were used by soaking them in the solution.

Aqueous extracts $\left(\mathrm{H}_{2} \mathrm{O}\right)$ 


\section{International Journal of Science and Research (IJSR) \\ ISSN (Online): 2319-7064}

Index Copernicus Value (2013): 6.14 | Impact Factor (2015): 6.391

Fifteen gm of Miswak powder was used; divided in three sterile Erlenmeyer flask, 5 gm each, to which 50, 20 and 15 $\mathrm{ml}$ of sterile deionized distilled water was added. The extracts soaked for two days at $6.6 \mathrm{C}^{\mathrm{o}}$ before the mixtures were centrifuged at $2,700 \mathrm{rpm}$ for 10 minutes. The supernatants were passed through a $90 \mathrm{~mm}$ membrane filter, the extract was prepared at 10,25 and 33.3 concentrations $(\mathrm{wt} / \mathrm{vol} \%)$ and stored at $24 \mathrm{C}^{\mathrm{o}}$.

\section{Methanol extracts (MeOH)}

Ten gm of Miswak powder was mixed with $100 \mathrm{ml}$ of $\mathrm{MeOH}$ in a sterile Erlenmeyer flask. Soxhlet extractor was used for an hour to separate the content. The dissolved Miswak powder was stored at $36.9 \mathrm{C}^{\circ}$ for $24 \mathrm{~h}$. The solid obtained was used after being dissolved in DMSO with a concentration of $1 \%$. That was prepared by mixing $99 \mathrm{ml}$ of normal saline with $1 \mathrm{ml}$ of DMSO. The DMSO was filtered from the mixture using a $90 \mathrm{~mm}$ membrane filter. Then the mixture was centrifuged at $5000 \mathrm{r} / \mathrm{m}$ for a minute.

\section{Antimicrobial susceptibility testing Toothpastes}

S.mutans was cultured in MHA. Then toothpastes drops were placed. Plates kept in a $37^{\circ} \mathrm{C}$ incubator for $24 \mathrm{~h}$.

\section{Aqueous extracts $\left(\mathrm{H}_{2} \mathrm{O}\right)$}

Miswak extract was platted on MHA as droplets using loops. The liquid supernatant was poured in holes on MHA. Disks were used by soaking them in the supernatant, and then they were placed on the surface of the plate. Plates were incubated in $37 \mathrm{C}^{\circ}$ for $24 \mathrm{~h}$.

\section{Methanol extracts (MeOH)}

Same procedure as aqueous extracts.

Experiments were repeated for both methanol and normal saline method.

\section{Results}

Four toothpastes were excluded for showing bacterial growth on BA and MCA. Crest toothpaste showed minimal effect against S.mutans. On the other hand, Aloedent showed no effect Figure (1).

First testing of Miswak, both the powder and the disks showed no effect against S.mutans. Microbial growth observed Figure (2).

Consecutive experiments were conducted after sterilization of Miswak.

Normal saline powder extract showed large inhibition zones on MHA; Figure (3). Moreover, the liquid supernatant of the normal saline method had obviously decreased the growth of S.mutans on MHA; Figure (4). However, the sterilized paper disks that were soaked in the supernatant of the normal saline method showed no effect in the second and third experiments; Figure $(5,6)$.

In the repeated tests, normal saline powder extract showed no effect with different concentrations; Figure (7) while $\mathrm{MeOH}$ extracts showed positive effect; Figure (8) and (9).

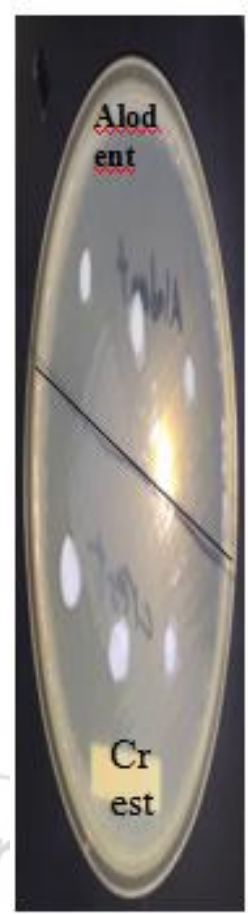

Figure 1: Tooth pastes effect on S.mutans on MHA.

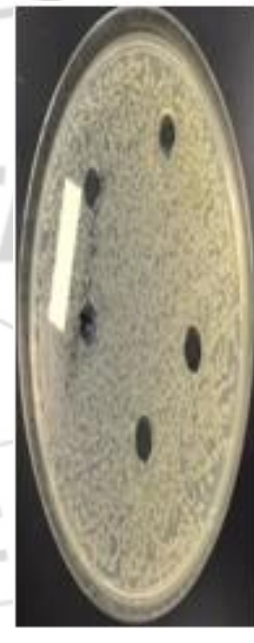

Figure 4:

Liquid

supernatant of normal saline,

decreased

growth of

S.mutans in

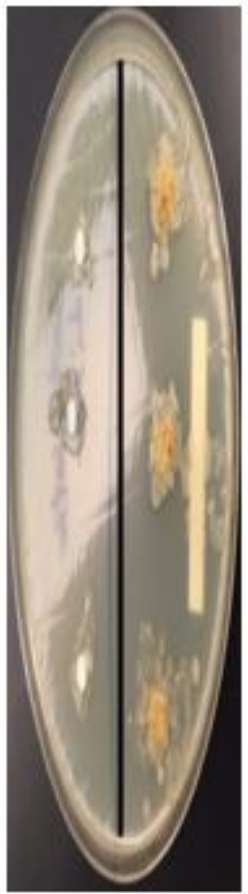

Figure 2:

Miswak, powder and disks on

S.mutans on

MHA in $1^{\text {st }}$ experiment.

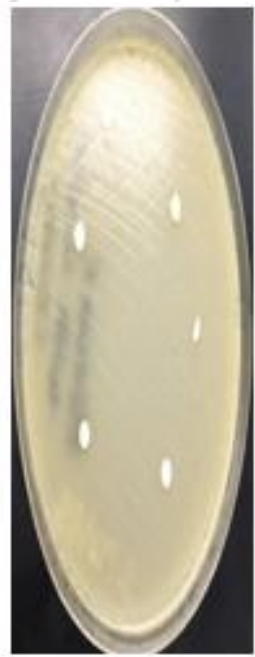

Figure 5: Soaked disks in normal saline supernatant showed no effect in $2^{\text {as }}$

experiment.

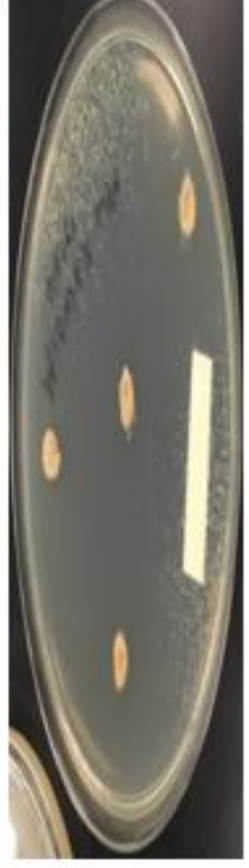

Figure 3: Normal saline extract effect on S.mutans, large inhibition zones recognized in $2^{\text {os }}$ experiment.

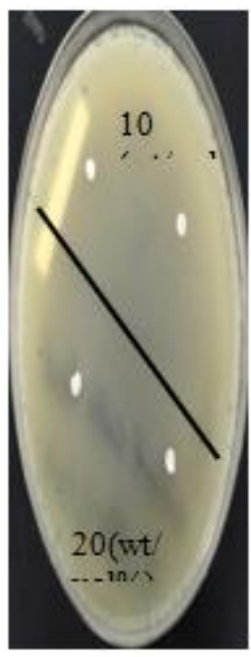

Figure 6: Soaked disks in normal saline supernatant showed no effect in $3^{\text {rd }}$ experiment. 


\section{International Journal of Science and Research (IJSR) ISSN (Online): 2319-7064}

Index Copernicus Value (2013): 6.14 | Impact Factor (2015): 6.391

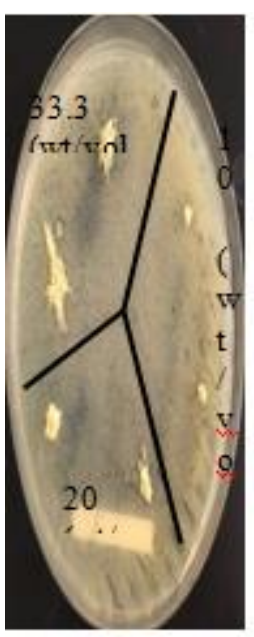

Figure 7: Normal saline extract with no effect on $\mathrm{S}$ mutans in

$3^{\text {rd }}$ experiment

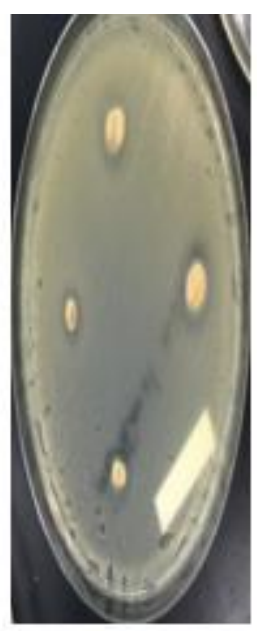

Figure 8: Inhibition zones were observed of $\mathrm{MeOH}$ extract on

S.mutans in $2^{\text {so }}$ experiment.

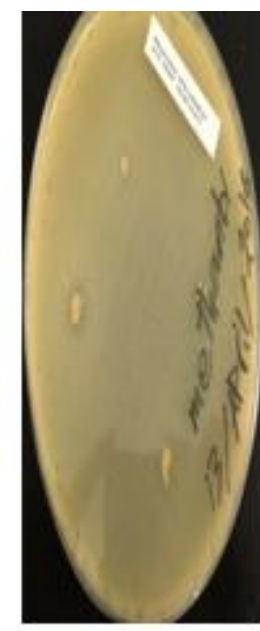

Figure 9: Inhibition zones were observed of $\mathrm{MeOH}$ extract on S.mutans in $3^{\text {so }}$ experiment.

\section{Discussion}

Four out of the six toothpastes were excluded for being contaminated. The reason for this was not clear, however, it might be due the manufacturer source. It is concluded that the bacteria, which grew in BA, was gram-positive. Some study showed the risk of contaminated toothpastes, which lead to transmission of cariogenic and periodontopathogenic species [17]. Therefore, random toothpastes samples further testing and notification of Suadi food and drug authority, is recommended. Some studies showed that some toothpastes can increase the bacteria in the oral cavity and referred the reason to the ingredients sodium saccharin and other sweeteners in toothpastes [18]. However, in this study, the effect of Crest toothpaste had minimal effect and AloeDent showed no effect. That can be attributed to the ingredients; Crest has sodium fluoride but AloeDent is fluoride free, so it is obvious that the toothpaste which has an antibacterial agent like fluoride is more effective[19,20]. Toothpaste minimal effect on the bacteria would conclude a strong recommendation for frequent oral hygiene and cleaning. Most of the cleaning is achieved by the mechanical action of the toothbrush, and not by the toothpaste[21].

These microbes can be considered as normal flora of the Miswak itself and this need further studies.

On the other hand, $\mathrm{MeOH}$ and normal saline Miswak extracts showed antibacterial activity the first time. The reasons for unsatisfactory results in the second round of the test could be explained or related to many factors; e.g. storage and grinding of the Miswak from farm beside some laboratory factors.

However, the $\mathrm{MeOH}$ extract showed an inhibition zone at both times. Most of the findings regarding of $\mathrm{MeOH}$ extract efficacy were consistent with other studies[22, 23]. In addition, what it should be taken into consideration is that the activity of Miswak is different in the oral cavity from its activity in vitro. So it is recommended to do further studies on the local population to see the effects of Miswak and toothpaste after using them. Moreover, further work needed for testing the effect of toothpastes and Miswak on normal and transient oral flora other microorganisms such as aggregatibacter actinomycetemcomitans, Eikenella corrodens, Fusobacterium nucleatum, Porphyromonas gingivalis and Prevotella intermedia.

\section{References}

[1] J. Loesche W. Role of Streptococcus mutans in Human Dental Decay. department of Oral Biology, School of Dentistry and Department of Microbiology and Immunology, School of Medicine, University of Michigan, Ann Arbor, Michigan 48109. 1986.

[2] Bagramian, Robert A., Franklin Garcia-Godoy, and Anthony R. Volpe. "The global increase in dental caries. A pending public health crisis." Am J Dent 22.1 (2009): 3-8.

[3] Islam, Barira, Shahper N. Khan, and Asad U. Khan. "Dental caries: from infection to prevention." Medical Science Monitor Basic Research 13.11 (2007): RA196RA203.

[4] United States Department of Health and Human Services (USDHHS). Oral Health in America: A Report of the Surgeon General. National Institute of Health, 2000.

[5] Simard F, andry R. Mouthrinses as an antibacterial adjunct $\mathrm{n}$ periodontal treatment. J Can Dent Assoc 1994;60(10):906-11

[6] Hamada S, D. Slade H. Biology, Immunology, and Cariogenicity of Streptococcus mutans. Department of Oral Biology, School of Dentistry, University of Colorado Health Sciences Center, Denver, Colorado 80262. 1980.

[7] Van Houte J. Microbiological predictors of caries risk. Adv Dent Res 1993: 7(2): 87-96.

[8] Loesche, W. J., J. Rowan, L. H. Straffon, and P. J. Loos. Association of Streptococcus mutans with human dental decay. Infect. Immun.1975.

[9] Lemos J, Quivey R , Koo H, Abranches J (2013) Streptococcus mutans: a new Gram-positive paradigm? Microbiology 159: 436-45. doi: 10.1099/mic.0.0661340 .

[10] Moeintaghavi A. In vitro Antimicrobial Comparison of Chlorhexidine, Persica Mouthwash and Miswak Extract. JCDP. 2012:147-152.

[11] Marsh P. Controlling the oral biofilm with antimicrobials. J Dent 2010; 38 Suppl 1:11-15.

[12] Hofer D, Meier A, Sener B, Guggenheim B, Attin T, Schmidlin PR. Biofilm reduction and staining potential of a $0.05 \%$ chlorhexidine rinse containing essential oils. Int J Dent Hyg 2011;9:60-67.

[13] Sih Mahanani E, fadhli Khamis M, Mochamad arie E, nabilah Mat rippin S, ahmad rajion Z. Antibacterial efficacy of Salvadora persica as a cleansing teeth towards Streptococcus mutans and Lactobacilli colonies. Dental journal (Maj Ked Gigi). Malaysia, Indonesia, 2012.

[14] Chaurasia A, Patil R, Nagar A. Miswak in oral cavity An update. Journal of Oral Biology and Craniofacial Research. 2013;3(2):98-101. 


\section{International Journal of Science and Research (IJSR) \\ ISSN (Online): 2319-7064}

Index Copernicus Value (2013): 6.14 | Impact Factor (2015): 6.391

[15] Khalessi A, Pack A, Thomson W, Tompkins G. An in vivo study of the plaque control efficacy of Persica ${ }^{\text {TM}}$ : a commercially available herbal mouthwash containing extracts of Salvadora persica. International Dental Journal. 2004;54(5):279-283.

[16] World Health Organization. Prevention of oral diseases, WHO, 1987.

[17] Schmidt J, Bux M, Filipuzzi-Jenny E, Kulik E, Waltimo $\mathrm{T}$, Weiger $\mathrm{R}$ et al. Influence of time, toothpaste and saliva in the retention of Streptococcus mutans and Streptococcus sanguinis on different toothbrushes. Journal of Applied Oral Science. 2014;22(3):152-158.

[18] Okpalugo J, Ibrahim K, Inyang U. Toothpaste formulation efficacy in reducing oral flora. Tropical Journal of Pharmaceutical Research. 2009;8(1).

[19]Bowden G. Effects of Fluoride on the Microbial Ecology of Dental Plaque. J DENT RES. 1990;69(2 suppl):653-659.

[20] Maria K, Geetha R. Effect Of Herbal And Fluoride Toothpaste On Streptococcus Mutans - A Comparative Study. Journal of pharmaceutical sciences and research. 2015;7(10):864-865.

[21] El-Ishaq A, Bello L, Aidi A. The Role Of Various Toothpaste In Reduction Of Bacteria Load In The Mouth. Extensive Journal of Applied Sciences. 2014;3(2):49-54.

[22] Al-Bayati F, Sulaiman K. In Vitro Antimicrobial Activity of Salvadora persica L. Extracts Against Some Isolated Oral Pathogens in Iraq. Turk J Biol. 2008;32:57-62.

[23] Popova C, Dosseva V, Panov V. Microbiology of Periodontal Diseases. A Review. Biotechnology \& Biotechnological Equipment. 2013;27(3):3754-3759. 